\title{
Robust $L_{p}-$ Norm Metric for BICM-OFDM Cognitive Radio Systems
}

\author{
Amir Nasri and Robert Schober \\ University of British Columbia, \{amirn, rschober\}@ece.ubc.ca \\ Invited Paper
}

\begin{abstract}
Cognitive radio (CR) systems make efficient use of the frequency spectrum by opportunistically exploiting unoccupied or under-utilized frequency bands. However, the frequency bands used by CR systems are expected to suffer from various forms of noise and interference with non-Gaussian distributions, such as narrowband and co-channel interference caused by the primary user and other CRs, respectively, and man-made impulsive noise. To mitigate the harmful effects of non-Gaussian noise and interference, we propose a robust $L_{p}$-norm metric for CR systems that employ the popular combination of bitinterleaved coded modulation (BICM) and orthogonal frequency division multiplexing (OFDM). For the considered CR system we provide an approximate upper bound and an asymptotic closed-form approximation for the bit error rate (BER). Our results show that the proposed $L_{p}$-norm metric can achieve large performance gains compared to the conventional $L_{2}$-norm metric in non-Gaussian noise and interference.
\end{abstract}

\section{INTRODUCTION}

Cognitive radio (CR) is a relatively new concept that enables more efficient utilization of spectral resources [1]. In a broad sense a CR is a smart radio capable of efficiently exploiting frequency bands which are temporally or spatially unoccupied or under-utilized by their licensed (primary) users. While primary users have priority in spectrum access, (secondary) CR users can use the available spectrum through opportunistic access as long as they do not interfere with primary users.

While most proposed CR systems employ traditional methods of signal detection designed for additive white Gaussian noise (AWGN), various forms of non-Gaussian noise and interference can be present in CR environments. Examples include the narrowband and co-channel interference caused by the primary user and other CR systems [2], respectively, and man-made impulsive noise [3] caused by e.g. by microwave ovens [4]. Therefore, the use of the $L_{2}$-norm metric (also referred to as the Euclidean distance metric) for signal detection, which is optimal for Gaussian noise, can result in significant performance losses in CR environments where non-Gaussian noise ${ }^{1}$ is dominant. This motivates the use of robust metrics that perform well for a large class of noises with (possibly) non-Gaussian distribution. Furthermore, these metrics may also have tunable parameters that can be adjusted to the possibly time-variant noise statistics. Important examples of such robust metrics in the literature include Huber's $M$-metric [5], Myriad and Meridian metrics [6], and the $L_{p}$-norm metric [7]. Among these metrics, the $L_{p}$-norm metric is particularly

\footnotetext{
${ }^{1}$ To simplify our notation, in this paper, "noise" refers to any additive impairment of the received signal, i.e., our definition of noise also includes what is commonly referred to as "interference".
}

interesting due to its low complexity and the ability to perform well in both heavy-tailed and short-tailed noise provided that the metric parameter $p$ is adjusted accordingly.

In this paper, we consider CR systems employing the popular combination of bit-interleaved coded modulation (BICM) and orthogonal frequency division multiplexing (OFDM). BICM-OFDM has been adopted by a number of recent standards [8] and is also a prime candidate for the air interface of future CR systems. Here, we propose a robust $L_{p}$-norm decoding metric for BICM-OFDM CR systems to mitigate the harmful effects of non-Gaussian impairments. We provide a tight approximate upper bound on the bit error rate (BER) of the resulting CR system. Furthermore, we derive a simple and easy-to-evaluate approximation for the asymptotic BER by analyzing the behavior of the upper bound at high signalto-noise ratios (SNRs). The asymptotic BER is obtained as a function of the metric parameter $p$ and therefore can be used for metric optimization. Simulation results confirm the validity of our analysis and show that the proposed $L_{p}$-norm metric is very effective in dealing with the harmful effects of non-Gaussian noise.

The rest of this paper is organized as follows. In Section II, the system model for the considered CR system is introduced. The approximate upper bound for the BER is derived in Section III and asymptotic BER expressions are obtained in Section IV. Simulation and numerical results are presented in Section V, and conclusions are drawn in Section VI.

\section{SySTEM MODEL}

In the following, we describe the signal and noise models for the considered BICM-OFDM CR system. For convenience, in this paper, all signals and systems are represented by their complex baseband equivalents.

\section{A. Signal Model}

The transmitter for the considered CR system consists of a BICM encoder and an OFDM modulator with $N$ sub-carriers. The BICM encoder comprises a convolutional encoder of rate $R_{c}$, an interleaver, and a memoryless mapper [9]. The codeword $\boldsymbol{c} \triangleq\left[c_{1}, c_{2}, \ldots, c_{m_{c} K_{c}}\right]$ of length $m_{c} K_{c}$ is generated by the convolutional encoder and is interleaved by the interleaver. The interleaved bits are broken up into blocks of $m_{c}$ bits, which are subsequently mapped to symbols $x_{k}$ from a constellation $\mathcal{X}$ of size $|\mathcal{X}| \triangleq M=2^{m_{c}}$ to form the transmit sequence $\boldsymbol{x} \triangleq\left[x_{1}, x_{2}, \ldots, x_{K_{c}}\right]$ of length $K_{c}$. We assume that one codeword spans $B$ OFDM symbols, i.e., $K_{c}=B N$, and that the length of the OFDM cyclic prefix exceeds the length of 
the channel impulse response. Furthermore, we assume that the channel changes independently from OFDM symbol to OFDM symbol, which can be achieved by frequency hopping. For example, the ECMA multi-band OFDM (MB-OFDM) ultrawideband (UWB) system employs interleaving and coding over $B=3$ (future versions of the standard may use up to $B=15$ ) frequency-hopped OFDM symbols [10]. Assuming perfect synchronization and OFDM demodulation, the received signal can be written as

$$
r_{k}=\sqrt{\gamma} h_{k} x_{k}+n_{k}, \quad 1 \leq k \leq K_{c},
$$

where $h_{k}$ and $n_{k}$ with $\mathcal{E}\left\{\left|h_{k}\right|^{2}\right\}=\mathcal{E}\left\{\left|n_{k}\right|^{2}\right\}=1$ are the fading gains and the noise variables, respectively, and $\gamma$ denotes the SNR. ${ }^{2}$ In this paper, we consider Rayleigh fading which implies that $h_{k}$ is a zero-mean complex Gaussian random variable (RV). Therefore, the fading gains $h_{k}$ can be expressed as $h_{k} \triangleq a_{k} e^{j \Theta_{k}}$, where $a_{k}$ and $\Theta_{k}$ are independent RVs. Specifically, $\Theta_{k}$ is uniformly distributed in $[-\pi, \pi)$ and $a_{k}$ is a positive real RV that follows a Rayleigh distibution. As customary in the literature, cf. e.g. [9], [11], [12], for our performance analysis we assume perfect interleaving, which means that $h_{k}$ and $n_{k}$ can be modeled as independent, identically distributed (i.i.d.) RVs and only their first order probability density functions (pdfs) are relevant. The i.i.d. assumption is justified for severely frequency selective channels and/or sufficiently large $B$.

\section{B. $L_{p}-$ Norm Metric}

Traditional BICM-OFDM systems employ an $L_{2}$-norm metric as branch metric for Viterbi decoding at the receiver. In contrast, here we consider Viterbi decoding with an $L_{p^{-}}$ norm branch metric that allows the CR system to adapt to the ambient noise environment by adjusting the metric parameter $p$. The branch metric for bit $i, 1 \leq i \leq m_{c}$, of symbol $x_{k}$ is given by

$$
\lambda_{i}\left(r_{k}, b\right) \triangleq \min _{x_{k} \in \mathcal{X}_{b}^{i}}\left\{\left|r_{k}-\sqrt{\gamma} h_{k} x_{k}\right|^{p}\right\}
$$

where $p$ is the adjustable metric parameter. Furthermore, $\mathcal{X}_{b}^{i}$ denotes the subset of all symbols in constellation $\mathcal{X}$ whose label has value $b \in\{0,1\}$ in position $i$.

\section{Noise Model}

In this subsection, we present two important non-Gaussian noise models that are frequently encountered in CR environments. In particular, we consider narrowband interference (NBI) and frequency-domain Gaussian mixture noise (GMN). These noise models are used in Section $\mathrm{V}$ for performance valuation of the proposed $L_{p}$-norm metric.

NBI: Consider a BICM-OFDM CR system with coding over $B$ different hopping frequencies. At hopping frequency $\mu, 1 \leq \mu \leq B$, the received frequency-domain signal is impaired by AWGN $\tilde{n}_{k, \mu}$ and $I_{\mu}$ Rayleigh-faded phase-shift

\footnotetext{
${ }^{2}$ In this paper, $\mathcal{E}_{x}\{\cdot\}$ denotes statistical expectation with respect to $x$. Furthermore, we use the notation $u \stackrel{\circ}{=}$ to indicate that $u$ and $v$ are asymptotically equivalent, and a function $f(x)$ is $o(g(x))$ if $\lim _{x \rightarrow 0} f(x) / g(x)=0$.
}

keying (PSK) NBI signals. The corresponding frequencydomain noise model is

$$
n_{k, \mu}=\sum_{i=1}^{I_{\mu}} g_{k, \mu}[i] b_{\mu}[i] \tilde{h}_{k, \mu}[i]+\tilde{n}_{k, \mu}, \quad 1 \leq k \leq N
$$

where $b_{\mu}[i]$ is the PSK symbol of the $i$ th interferer at the $\mu$ th hopping frequency affecting the set $\mathcal{N}_{\mu, i}$ of sub-carriers via $g_{k, \mu}[i] \triangleq \exp \left[-j \pi(N-1)\left(k+f_{\mu, i} / \Delta f_{s}\right) / N+\phi_{\mu, i}\right] \sin [\pi(k+$ $\left.\left.f_{\mu, i} / \Delta f_{s}\right)\right] / \sin \left[\pi\left(k+f_{\mu, i} / \Delta f_{s}\right) / N\right]$ [13]. Here, $f_{\mu, i}$ and $\phi_{\mu, i}$ denote the frequency and phase of the $i$ th interferer at hopping frequency $\mu$ relative to the user, respectively, and $\Delta f_{s}$ is the OFDM sub-carrier spacing. Finally, we assume that no subcarrier is affected by two narrowband interferers at a given hopping frequency, i.e., $\mathcal{N}_{\mu, i_{1}} \cap \mathcal{N}_{\mu, i_{2}}=\emptyset, i_{1} \neq i_{2}$. The pdf for this general interference scenario is given by

$$
\begin{aligned}
p_{n}\left(n_{k}\right)=\sum_{\mu=1}^{B} & \sum_{i=1}^{I_{\mu}} \sum_{k \in \mathcal{N}_{\mu, i}} \frac{c_{0}}{\pi \sigma_{\mu, i, k}^{2}} \exp \left(-\frac{\left|n_{k}\right|^{2}}{\sigma_{\mu, i, k}^{2}}\right) \\
& +\frac{c_{1}}{\pi \sigma_{\tilde{n}}^{2}} \exp \left(-\frac{\left|n_{k}\right|^{2}}{\sigma_{\tilde{n}}^{2}}\right),
\end{aligned}
$$

where $\sigma_{\tilde{n}}^{2}$ denotes the variance the AWGN, $c_{0} \triangleq 1 /(B N)$, $c_{1} \triangleq 1-\sum_{\mu=1}^{B} \sum_{i=1}^{I_{\mu}}\left|\mathcal{N}_{\mu, i}\right| /(B N)$, and $\sigma_{\mu, i, k}^{2} \triangleq$ $\left|g_{k, \mu}[i]\right|^{2} \mathcal{E}\left\{\left|\tilde{h}_{k, \mu}[i]\right|^{2}\right\}+\sigma_{\tilde{n}}^{2}$. For future reference, we denote the ratio of the total NBI variance and the AWGN variance by $\kappa$, cf. Section VI.

GMN: The frequency-domain GMN model can be used to model the combined effect of AWGN and frequency-hopped Rayleigh faded narrowband interferers (e.g. Bluetooth signals) or narrowband man-made impulsive noise. Denoting the the probability that the interferer or the impulsive noise affects a sub-carrier of the $\mathrm{CR}$ by $\epsilon$, we can express the pdf of the corresponding frequency-domain noise as

$$
p_{n}\left(n_{k}\right)=\frac{(1-\epsilon)}{\pi \sigma_{g}^{2}} \exp \left(-\frac{\left|n_{k}\right|^{2}}{\sigma_{g}^{2}}\right)+\frac{\epsilon}{\pi \kappa \sigma_{g}^{2}} \exp \left(-\frac{\left|n_{k}\right|^{2}}{\kappa \sigma_{g}^{2}}\right),
$$

where $\sigma_{g}^{2}$ is variance of the frequency-domain Gaussian background noise and $\kappa$ is the ratio of the variances of the background noise and the interference/impulsive noise.

\section{APPROXIMATE UPPER BOUND FOR BER}

In this section, we provide a tight approximate upper bound on the BER performance of the considered CR system in nonGaussian noise environments. In particular, for a CR system employing a convolutional code of rate $R_{c}=k_{c} / n_{c}\left(k_{c}\right.$ and $n_{c}$ are integers) the union bound for the BER is given by [9]

$$
P_{b} \leq \frac{1}{k_{c}} \sum_{d=d_{f}}^{\infty} w_{c}(d) P_{e}(\boldsymbol{c}, \hat{\boldsymbol{c}})
$$

where $\boldsymbol{c}$ and $\hat{\boldsymbol{c}}$ are two distinct code sequences with Hamming distance $d$ that differ only in $l \geq 1$ consecutive trellis states, $w_{c}(d)$ denotes the total input weight of error events at Hamming distance $d$, and $d_{f}$ is the free distance of the code. $P_{e}(\boldsymbol{c}, \hat{\boldsymbol{c}})$ is the pairwise error probability (PEP), i.e., the 
probability that the decoder chooses code sequence $\hat{\boldsymbol{c}}$ when code sequence $\boldsymbol{c} \neq \hat{\boldsymbol{c}}$ is transmitted. Invoking the approximate expurgated bound from [9], the PEP can be expressed as

$P_{e}(\boldsymbol{c}, \hat{\boldsymbol{c}})=\frac{1}{2 \pi j} \int_{c-j \infty}^{c+j \infty^{\infty}} \prod_{k=1}^{d}\left(\frac{1}{m_{c} 2^{m_{c}}} \sum_{i=1}^{m_{c}} \sum_{b=0}^{1} \sum_{x_{k} \in \mathcal{X}_{b}^{i}} \Phi_{\Delta}(s)\right) \frac{\mathrm{d} s}{s}$

where $c$ is a small positive constant that lies in the region of convergence of the integrand. Furthermore, $\Phi_{\Delta}(s) \triangleq$ $\mathcal{E}\left\{e^{-s \Delta\left(x_{k}, z_{k}\right)}\right\}$ is the moment generating function (MGF) of the metric difference

$$
\Delta\left(x_{k}, z_{k}\right) \triangleq\left|r_{k}-\sqrt{\gamma} h_{k} z_{k}\right|^{p}-\left|r_{k}-\sqrt{\gamma} h_{k} x_{k}\right|^{p}
$$

where $z_{k}$ is the nearest neighbor of $x_{k}$ in $\mathcal{X}_{\bar{b}}^{i}$ with $\bar{b}$ being the bit complement of $b$. Exploiting (1), (8) can be simplified to

$$
\Delta\left(x_{k}, z_{k}\right)=y_{k}-\left|n_{k}\right|^{p},
$$

where $y_{k} \triangleq\left|\sqrt{\gamma} h_{k} e_{k}+n_{k}\right|^{p}$ and $e_{k} \triangleq x_{k}-z_{k} \triangleq d_{x z} e^{j \Theta_{d}}$ with $d_{x z}>0$.

The MGF $\Phi_{\Delta}(s)$ can be obtained by numerical integration and subsequently be used for calculation of the approximate upper bound (6). Thereby, the integral in (7) can be efficiently evaluated numerically using a Gauss-Chebyshev quadrature rule, cf. [14]. We note that (6) is only an approximate upper bound since (7) is not a true upper bound [15].

\section{ASYMPTOTIC ANALYSis}

In this section, we analyze the asymptotic behavior of the approximate upper bound in (6) for $\gamma \rightarrow \infty$. For this purpose, it is convenient to first rewrite the PEP as

$$
P_{e}(\boldsymbol{c}, \hat{\boldsymbol{c}})=\frac{1}{2 \pi j} \int_{c-j \infty}^{c+j \infty} \mathcal{E}_{n_{k}}\left\{\Phi\left(s \mid n_{k}\right)\right\} \frac{\mathrm{d} s}{s},
$$

with

$$
\Phi\left(s \mid n_{k}\right)=\prod_{k=1}^{d}\left(\frac{1}{m_{c} 2^{m_{c}}} \sum_{i=1}^{m_{c}} \sum_{b=0}^{1} \sum_{x_{k} \in \mathcal{X}_{b}^{i}} \Phi_{\Delta}\left(s \mid n_{k}\right)\right),
$$

where

$$
\Phi_{\Delta}\left(s \mid n_{k}\right) \triangleq \mathcal{E}\left\{e^{-s \Delta\left(x_{k}, z_{k}\right)} \mid n_{k}\right\}=e^{-s\left|n_{k}\right|^{p}} \Phi_{y}(s),
$$

For the last equality we have used (9) and the definition $\Phi_{y}(s) \triangleq \mathcal{E}\left\{e^{-s y_{k}}\right\}$. For $\gamma \rightarrow \infty$, the pdf $f_{y}\left(y_{k}\right)$ of $y_{k}$ can be expressed as [16]

$$
f_{y}\left(y_{k}\right)=\frac{2}{p\left(\gamma d_{x z}^{2}\right)} y_{k}^{\frac{2}{p}-1}+o\left(\gamma^{-1}\right) .
$$

Therefore, the asymptotic MGF $\Phi_{y_{k}}(s)$ can be obtained as the Laplace transform of $f_{y}\left(y_{k}\right)$ as

$$
\Phi_{y}(s)=\frac{2}{p\left(\gamma d_{x z}^{2}\right)} \Gamma(2 / p) s^{-\frac{2}{p}}+o\left(\gamma^{-1}\right) .
$$

Applying (14) in (12) yields

$$
\Phi_{\Delta}\left(s \mid n_{k}\right)=\frac{2 e^{-s\left|n_{k}\right|^{p}}}{\left(\gamma d_{x z}^{2}\right)} \Gamma(2 / p) s^{-\frac{2}{p}}+o\left(\gamma^{-1}\right) .
$$

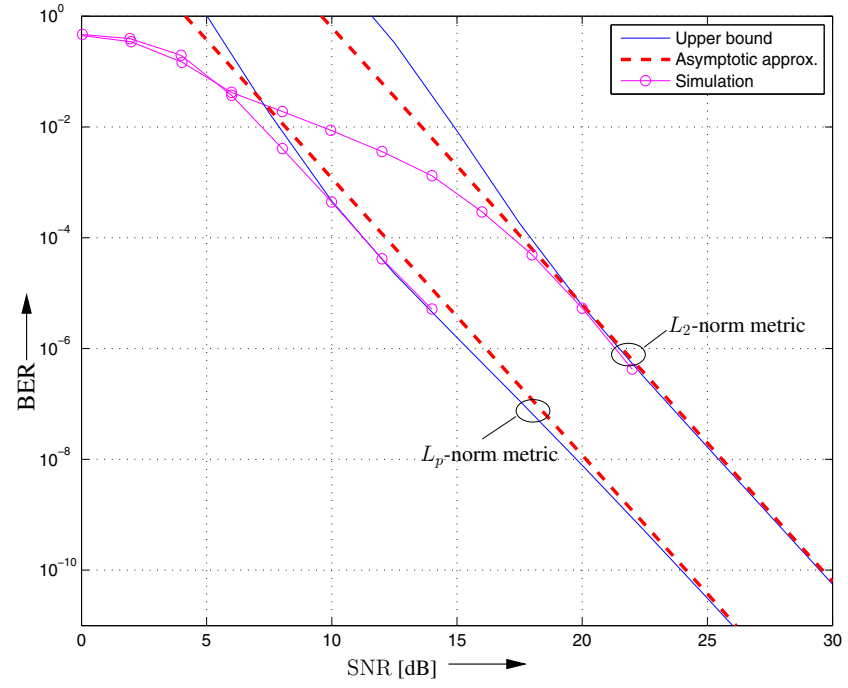

Fig. 1. BER of BICM-OFDM CR system with $B=5$ and $N=128$ impaired by NBI ( 5 equal power, sub-carrier-centered NBI signals, $I_{\mu}=1$, $1 \leq \mu \leq 5, \kappa=40)$ vs. SNR $\gamma$.

We can now obtain $\Phi\left(s \mid n_{k}\right)$ from (11) in (15) as

$$
\begin{gathered}
\Phi\left(s \mid n_{k}\right)=2^{d} X(d) \gamma^{-d} e^{-s \sum_{k=1}^{d}\left|n_{k}\right|^{p}}(\Gamma(2 / p))^{d} s^{-2 d / p} \\
+o\left(\gamma^{-d}\right)
\end{gathered}
$$

where

$$
X(d) \triangleq\left(\frac{1}{m_{c} 2^{m_{c}}} \sum_{i=1}^{m_{c}} \sum_{b=0}^{1} \sum_{x_{k} \in \mathcal{X}_{b}^{i}} \frac{1}{d_{x z}^{2}}\right)^{d}
$$

The PEP can be calculated from (16) and (10) as

$$
P_{e}\left(\boldsymbol{c}, \hat{\boldsymbol{c}} \mid n_{k}\right)=2^{d} X(d) \gamma^{-d} \frac{(\Gamma(2 / p))^{d}}{p^{d} \Gamma(2 d / p+1)} M_{n}(d, p),
$$

where the generalized noise moments $M_{n}(d, p)$ are defined as

$$
M_{n}(d, p) \triangleq \mathcal{E}_{n_{k}}\left\{\left(\sum_{k=1}^{d}\left|n_{k}\right|^{p}\right)^{2 d / p}\right\}
$$

The generalized noise moments $M_{n}(d, p)$ can be obtained in closed-form using a similar approach as in [17] or can be efficiently calculated using Monte-Carlo simulation.

Based on (18) and (6) a closed-form approximation for the asymptotic BER $P_{b} \stackrel{\circ}{=} \frac{w_{c}\left(d_{f}\right)}{k_{c}} P_{e}(\boldsymbol{c}, \hat{\boldsymbol{c}})$ can be obtained as

$$
P_{b} \doteq \frac{w_{c}\left(d_{f}\right) X\left(d_{f}\right) 2^{d_{f}}(\Gamma(2 / p))^{d_{f}}}{k_{c} p^{d_{f}} \Gamma\left(2 d_{f} / p+1\right)} M_{n}\left(d_{f}, p\right) \gamma^{-d_{f}}
$$

where $d_{f}$ denotes the minimum free distance of the convolutional code. The closed-form expression (20) reveals that the $L_{p}$-norm metric achieves a diversity gain of $d_{f}$ regardless of which $p$ is employed. However, as will be shown in the next section, optimizing $p$ results in significant coding gains. 


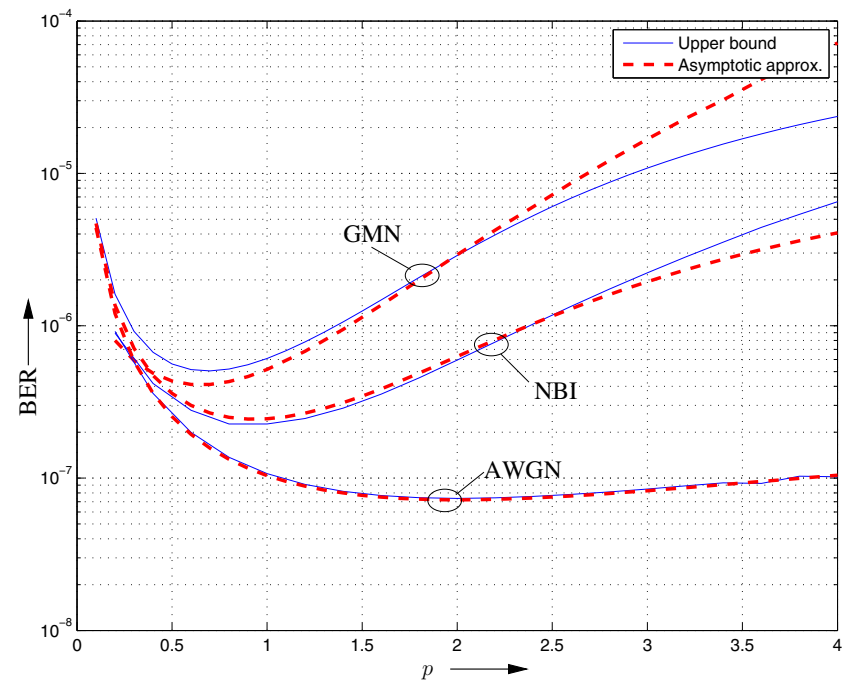

Fig. 2. BER of BICM-OFDM CR system $(N=64, B=5$, SNR $=16$ dB) impaired by different types of noise vs. $p$. NBI: 25 equal power, subcarrier-centered NBI signals, $I_{\mu}=5,1 \leq \mu \leq 5, \kappa=4$. GMN: $\epsilon=0.1$, $\kappa=10$.

\section{Numerical and Simulation Results}

In this section, we verify the analytical results presented in Sections III and IV with computer simulations. For all results shown we consider a BICM-OFDM CR system with $N=64$ or $N=128,4-\mathrm{PSK}$, and a code with rate $R_{c}=3 / 4$ obtained through puncturing of the standard rate- $1 / 2$ convolutional code with generator polynomials $[133,171]$ (octal representation). The parameters for the considered types of noise are specified in the captions of the figures.

Fig. 1 shows the BER of the considered CR system in the presence of NBI vs. SNR. Results for the conventional $L_{2}$-norm metric and the proposed $L_{p}$-norm metric with optimal $p=0.385$ are depticted. As can be observed, for sufficiently high SNR the derived approximate upper bound and asymptotic approximation for the BER agree well with the computer simulation results corroborating our analysis in Sections III and IV. Fig. 1 further shows that the proposed $L_{p}$-norm metric is very effective in mitigating the harmful effects of NBI and achieves a performance gain of more than $5 \mathrm{~dB}$ compared to the $L_{2}$-norm metric at high SNRs.

To further investigate the dependence of the BER on the metric parameter $p$, in Fig. 2, we show the BER of NBI, GMN, and AWGN as functions of $p$ for SNR $=16 \mathrm{~dB}$. For the sake of clarity, we only show the approximate upper bound and the asymptotic approximation in Fig. 2. We note, however, that these results were confirmed by computer simulations. Note that the approximate upper bound is not necessarily an upper bound on the proposed asymptotic approximation. Fig. 2 shows that while for AWGN $p=2$ is optimal as expected, for heavy-tailed types of noise such as NBI and GMN the optimal $p$ is smaller than two. The excellent agreement between the upper bound and the asymptotic approximation, especially at low BERs, suggests that the $L_{p}$-norm metric can be optimized based on the relatively simple expression (20) for the asymptotic approximation instead of the more cumbersome upper bound.

\section{CONCLUSIONS}

In this paper, we have proposed an $L_{p}$-norm metric for BICM-OFDM CR systems operating in the presence of nonGaussian noise. We have derived an approximate upper bound and an accurate asymptotic approximation for the BER of the considered system. These analytical results can be used for optimization of the metric parameter $p$. Simulation results have confirmed the validity of the provided analytical results and have shown the effectiveness of the proposed $L_{p}$-norm metric in mitigating the harmful effects of non-Gaussian noise in $\mathrm{CR}$ systems.

\section{REFERENCES}

[1] J. Mitola. Cognitive Radio: an Integrated Agent Architecture for Software Defined Radio. Ph.d. thesis, KTH Royal Inst. of Tech., Stockholm, Sweden, 2000.

[2] K. Watanabe, K. Ishibashi, and R. Kohno. Performance of Cognitive Radio Technologies in the Presence of Primary Radio Systems. In Proc. IEEE Intern. Symp. Person., Indoor and Mobile Radio Commun. (PIMRC), pages 1-5, September 2007.

[3] D. Middleton. Statistical-physical Models of Man-made Radio Noise Parts I and II. U.S. Dept. Commerce Office Telecommun., April 1974 and 1976.

[4] T. Taher, M. Misurac, J. LoCicero, and D. Ucci. Microwave Oven Signal Interference Mitigation For Wi-Fi Communication Systems. In Proc. IEEE Consumer Commun. and Networking Conf. (CCNC), pages 67-68, January 2008.

[5] P. Huber. Robust Statistics. Wiley, New York, 1981.

[6] T. Aysal and K. Barner. Meridian Filtering for Robust Signal Processing. IEEE Trans. Signal Processing, 55:39493962, August 2007.

[7] G. Shevlyakov and K. Kim. Robust Minimax Detection of a Weak Signal in Noise With a Bounded Variance and Density Value at the Center of Symmetry. IEEE Trans. Inform. Theory, 52:12061211, March 2006.

[8] H. Bölcskei. MIMO-OFDM Wireless Systems: Basics, Perspectives, and Challenges. IEEE Wireless Commun., 13:31-37, August 2006.

[9] G. Caire, G. Taricco, and E. Biglieri. Bit-Interleaved Coded Modulation. IEEE Trans. Inform. Theory, 44:927-946, May 1998.

[10] ECMA. Standard ECMA-368: High Rate Ultra Wideband PHY and MAC Standard. [Online] http://www.ecmainternational.org/publications/standards/Ecma-368.htm, December 2005.

[11] P.-C. Yeh, S. Zummo, and W. Stark. Error Probability of Bit-Interleaved Coded Modulation in Wireless Environments. IEEE Trans. Veh. Technol., 55:722-728, March 2006.

[12] D. Rende and T. Wong. Bit-Interleaved Space-Frequency Coded Modulation for OFDM Systems. IEEE Trans. Wireless Commun., 4:2256-2266, September 2005.

[13] A. Coulson. Bit Error Rate Performance of OFDM in Narrowband Interference with Excission Filtering. IEEE Trans. Wireless Commun., 5:2484-2492, September 2006.

[14] E. Biglieri, G. Caire, G. Taricco, and J. Ventura-Traveset. Computing Error Probabilities over Fading Channels: a Unified Approach. European Trans. Telecommun., 9:15-25, Jan./Feb. 1998.

[15] V. Sethuraman and B. Hajek. Comments on "Bit-Interleaved Coded Modulation”. IEEE Trans. Inform. Theory, 52:1795-1797, April 2006.

[16] A. Nasri and R. Schober. Robust $L_{p}$-Norm Metric for BICM-Based Cognitive Radio Systems. To be submitted to IEEE Trans. on Commun., 2009.

[17] A. Nasri, A. Nezampoor, and R. Schober. Adaptive $L_{p^{-}}$ Norm Diversity Combining in Non-Gaussian Noise and Interference. Submitted to IEEE Trans. on Wireless Commun. [Online] http://www.ece.ubc.ca/ amirn/TW-08.pdf, 2008. 